\title{
Análisis de la sequía en la subcuenca del río Bonito mediante el índice de precipitación estandarizado
}

\author{
Carlos Manuel Veroy Reyes, ${ }^{1}$ Raúl Orlando Valerio Martínez, ${ }^{2}$ \\ Carmen Leticia Crespo, ${ }^{3}$ Gilda Elena Aguilar ${ }^{4}$
}

\section{RESUMEN}

El presente artículo detalla el trabajo realizado para analizar la sequía en la subcuenca del rio Bonito mediante el uso del índice de precipitación estandarizado y los registros de precipitación de las estaciones meteorológicas aledañas a la zona de estudio, a partir de lo cual se encontraron todos los eventos secos y húmedos entre los años 1958-2012.

Además, se presentan los gráficos de máximos y mínimos promedio de precipitación, la relación entre años del fenómeno El Niño (ENOS) y eventos secos, mapas geoespaciales de las estaciones meteorológicas con su índice de precipitación para años y meses claves de estudio, tales como la del fenómeno de El Niño de 19861988 , huracán Mitch y periodos secos que han afectado a los pobladores de la zona.

Se recolectaron datos de precipitación de 7 estaciones meteorológicas para encontrar las ocurrencias de eventos secos y eventos húmedos en la zona de la subcuenca del río Bonito durante todo el periodo de estudio 1959-2012. Mediante la transformación de valores de precipitación mensuales a valores de IPE (SPI-1, SPI12, etc.), los datos se ajustan a una distribución de probabilidad normal. De dichos resultados se recogen la presencia de los eventos en determinado mes, su caracterización como evento seco o húmedo con su grado de influencia, ya sea leve, moderada o extrema. Por último, se generan los mapas correspondientes a los períodos de mayor influencia encontrada.

Se analizaron los resultados totales y se identificaron 3 períodos de severa sequía 1986-1988, 1995, 2009-2010. Igualmente, se presentó un período de alta precipitación desde 2006 al 2008. Se identificaron los períodos de bajo nivel en los máximos

\footnotetext{
${ }^{1}$ Universidad Nacional Autónoma de Honduras. Facultad de Ciencias, Escuela de Matemáticas y Ciencias de la Computación. Correo electrónico: carlos.veroy@gmail.com

${ }^{2}$ Universidad Nacional Autónoma de Honduras. UNAH-TEC-Danlí. Sección de Matemáticas. Correo Electrónico: raulvaleriom@yahoo.com

${ }^{3}$ Universidad Nacional Autónoma de Honduras. Centro Universitario Regional del Litoral Atlántico (CURLA). Sección de Matemáticas.

${ }^{4}$ Universidad Nacional Autónoma de Honduras. Centro Universitario Regional del Litoral Atlántico (CURLA). Sección de Química.
} 
promedio de precipitación, donde dichos períodos de larga prolongación van desde 1983 hasta 1988 y de 1991 a 1995, que fueron períodos donde se presentó el fenómeno de EI Niño en la mayoría de los años. Mediante el análisis del SPI-12, en el período de 1975 a 2012, se caracterizaron 18 diferentes años como secos, es decir, se presentaron más eventos secos extremos que eventos húmedos extremos a lo largo de un año.

Palabras clave: sequía, IPE, precipitación, índice de precipitación estandarizado, estación meteorológica, fenómeno de El Niño, fenómeno La Niña, huracán Mitch, evento seco y evento húmedo.

\section{ABSTRACT}

The present work consisted in analyzing the drought of Río Bonito sub basin using standardized precipitation index and registers of pluviosity of meteorological stations close to the study zone, being found drought and wet events between 1958 and 2012.

It also shows a graph of maximum and minimum average precipitation, relation between EI Niño (ENOS) and drought events, geospatial maps of meteorological stations with their precipitation index to key years and months of study, such as El Niño 1986-1998, Mitch hurricane, and drought periods affected to inhabitants of zone.

Precipitation data from seven weather stations were collected to find the occurrences of dry events and wet events in the area of the Rio Bonito subbasin throughout the period 1959 -. 2012 By transforming values of monthly precipitation values IPE (SPI1 , SPI-12, etc.), the data fit a normal probability distribution. From those results we analyze the presence of events in a given month, its characterization as dry or wet event with their degree of influence: mild, moderate or extreme. Finally, maps are generated for most influenced periods.

The overall results were analyzed and 3 periods of severe drought from 1986 to 1988 , 1995, 2009-2010 were identified. Similarly, a period of high precipitation occurred from 2006 to 2008. Long periods of low-level maximum average precipitation were identified, ranging from 1983 to 1988 and 1991 to 1995. El Niño was present in most of these cases. By analysis of the SPI-12 in the period 1975 to 2012, there were characterized 18 different years as drought years, that is, the extreme dry events number was greater than wet events number over one calendar year.

Keywords: Drought, SPI, Precipitation, Standardized precipitation index, Meteorological stations, La Niña, El Niño, Mitch hurricane, Dry event and Wet event. 


\section{INTRODUCCIÓN}

La ciudad de La Ceiba se abastece de agua en parte de la subcuenca del río Bonito y se encuentra sujeta, al igual que todas las cuencas y subcuencas de Honduras, a situaciones adversas que pueden ser originadas por el cambio climático. En consecuencia, tomando en cuenta que la mayoría de las subcuencas carecen de un monitoreo adecuado, diferentes tipos de desastres naturales pueden afectar a esta ciudad (como la sequía), por lo tanto se beneficiaría de estudios que generen datos de monitoreo con los cuales sea posible hacer una mitigación más efectiva sobre los efectos adversos del cambio climático si se tiene un monitoreo de alerta oportuna, que en este caso se refiere a la sequía.

Debido a lo mencionado anteriormente, se tuvo la necesidad de encontrar métodos para poder identificar épocas secas y épocas lluviosas en la zona, con el fin de encontrar los distintos tipos de sequía que afectan a la subcuenca del río Bonito. Así, el método utilizado en esta investigación es el índice de precipitación estandarizado (SPI por sus siglas en inglés), desarrollado por Mckee en el año 1993, con el fin de mejorar la detección temprana del inicio de una sequía y su monitoreo.

El índice SPI es una herramienta simple, fácil de calcular y estadísticamente relevante y significativa, que permite medir en distintas escalas temporales a la sequía; estas escalas de tiempo reflejan el impacto de la sequía sobre la disponibilidad de los diferentes recursos de agua. Una de sus características más interesantes es que asigna un valor numérico a las precipitaciones, el cual se puede comparar con valores de todas las regiones, incluso con climas marcadamente diferentes. Es igual de eficaz para analizar tanto los periodos secos como los periodos húmedos y tiene como único parámetro de entrada a la precipitación.

El cálculo SPI para cualquier ubicación se basa en el registro de precipitación a largo plazo para un período deseado. Este registro a largo plazo se ajusta a una distribución de probabilidad, que se transforma, entonces, en una distribución normal; de modo que la media de SPI para la ubicación y el periodo deseado es cero (Edwards y Mckee, 1997).

\section{DESCRIPCIÓN DEL PROBLEMA}

Honduras tiene muchos problemas debido a la falta de información relevante con respecto a las sequía de diversas zonas, en particular de la subcuenca del río Bonito, ya que esta abastece de agua a gran parte de la población de la ciudad de La Ceiba y sus alrededores. 
Ante esta problemática, se necesita realizar un análisis de la sequía, definida como la ausencia de pluviavilidad, que es uno de los desastres que afecta a los seres humanos y recursos naturales; por eso en Honduras se han realizado estudios similares sobre la sequía en la subcuenca del río Guacerique y del río Choluteca.

Además de la relevante importancia que tiene este tema para el país, ya que el análisis de la sequía servirá para generar datos que resuman la situaciones adversas a las cuales los pobladores de la zona están sujetos y para identificar fenómenos naturales ocurridos a lo largo de los años, esta investigación se enmarca en el cuarto eje de investigación: Ambiente, biodiversidad y desarrollo, que forma parte del tema prioritario número 11: Cambio climático y vulnerabilidad.

\section{OBJETIVOS}

Objetivo general

Determinar el índice de precipitación estandarizado (IPE) de la subcuenca del río Bonito, en La Ceiba, para identificar los niveles de sequía y la aplicabilidad del IPE para la zona de estudio.

Objetivos específicos

1. Sintetizar y normalizar los datos de precipitación de las estaciones meteorológicas aledañas a la zona.

2. Calcular el valor numérico del IPE.

3. Identificar las épocas secas y lluviosas en los últimos años.

4. Realizar gráficos de máximos y mínimos promedio de precipitación a lo largo de los años.

5. Realizar comparaciones entre épocas secas y los años en los cuales se presentó el fenómeno de El Niño.

6. Demostrar espacialmente la variabilidad de la sequía en la zona del río Bonito, mediante el software ArcGIS.

\section{METODOLOGÍA}

La investigación es de tipo cuantitativa, pues trata un problema que se relaciona con las ciencias físico-naturales. Para el logro de los objetivos se recolectaron datos numéricos de precipitación y se aplicó una distribución de probabilidad estadística para la obtención de resultados. 
Es una investigación exploratoria, ya que se necesita tener una perspectiva amplia del problema de sequía en la zona del río Bonito, identificando las variables que están influyendo en el problema, razón por la que esta investigación se centró en la variable precipitación mensual.

También es una investigación no experimental, según la temporalización, para lo que se utilizó el método longitudinal, ya que se recogieron datos en periodos de tiempo específicos (registros de precipitación) y se hicieron inferencias con respecto a los resultados obtenidos, sus cambios y consecuencias.

La población son las estaciones meteorológicas del país, ya que de estas se obtuvieron los registros de precipitación para este estudio. La muestra fue de 7 estaciones, porque están cerca de la zona de estudio y por eso no se necesitó hacer uso de herramientas estadísticas para este cálculo.

Para la operacionalización de la variable precipitación, se determinaron los siguientes conceptos:

\section{Variable}

Precipitación

\section{Definición conceptual}

La precipitación es cualquier forma de hidrometeoro que cae de la atmósfera y llega a la superficie terrestre.

\section{Definición operacional}

El pluviómetro es un instrumento que se emplea en las estaciones meteorológicas para la recogida y medición de la precipitación.

La cantidad de agua caída se expresa en milímetros de altura. El diseño básico de un pluviómetro consiste en una abertura superior (de área conocida) de entrada de agua al recipiente, que luego es dirigida a través de un embudo hacia un colector donde se recoge y puede medirse visualmente con una regla graduada o mediante el peso del agua depositada.

Normalmente la lectura se realiza cada 12 horas. Un litro caído en un metro cuadrado alcanzaría una altura de 1 milímetro. 
En resumen, la metodología efectuada para los propósitos de esta investigación consistió en lo siguiente:

1. Identificar la zona de estudio (ver mapa 1).

Mapa 1. Área subcuenca río Bonito y estaciones meteorológicas

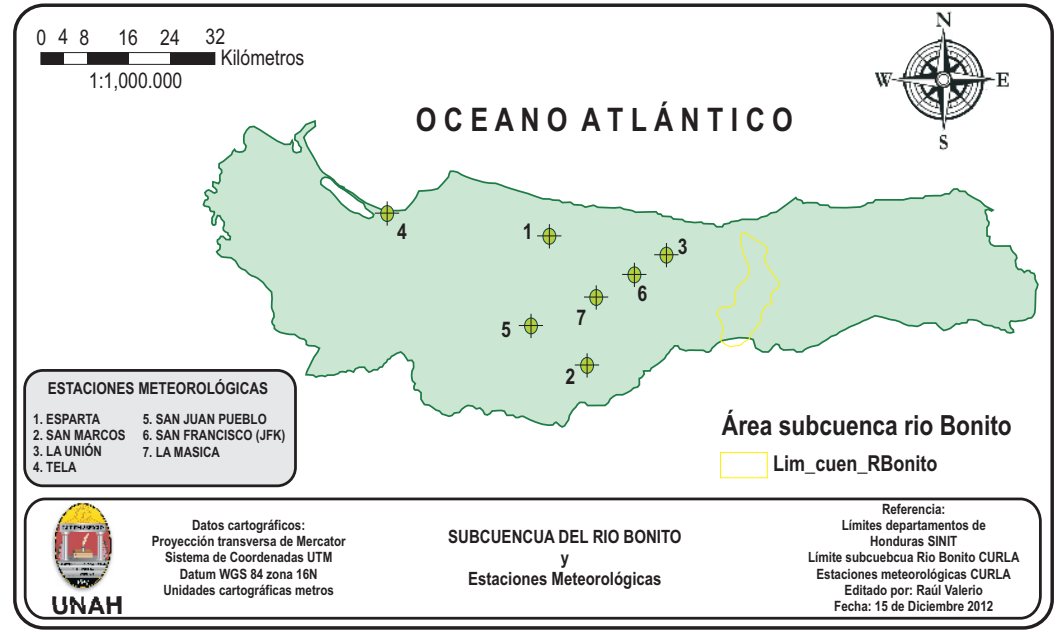

2. Se recolectaron datos de precipitación de las 7 estaciones meteorológicas aledañas a la zona: La Unión, Tela, San Juan Pueblo, San Francisco, San Marcos, La Masica y Esparta. Estas bases de datos fueron proporcionadas por SERNA, Servicio Meteorológico Nacional e Instituto Hondureño de Ciencias de La Tierra. Los datos faltantes fueron completados para tener una base de datos óptima y así garantizar la convergencia del software. En el cuadro 1 se presentan las estaciones meteorológicas con su ubicación en unidades UTM.

Cuadro 1. Estaciones meteorológicas y períodos de tiempo

\begin{tabular}{|l|c|c|c|}
\hline \multicolumn{1}{|c|}{ Estación } & Serie de tiempo & X & Y \\
\hline Esparta & $1972-1991$ & 481223 & 1740723 \\
\hline La Masica & $1986-2002$ & 489282 & 1729503 \\
\hline La Unión & $1972-2012$ & 502143 & 1737335 \\
\hline San Francisco (JFK) & $1980-2012$ & 496547 & 1733556 \\
\hline San Juan Pueblo & $1972-1999$ & 477368 & 1724350 \\
\hline San Marcos & $1979-2012$ & 487816 & 1716907 \\
\hline Tela & $1958-2008$ & 443587 & 1743950 \\
\hline
\end{tabular}

Fuente: Secretaría de Recursos Naturales (SERNA), registros de precipitación, 2012 
3. Se seleccionó el periodo de 1959-2012 y se calculó el IPE-12 en toda la subcuenca del río Bonito, luego se hizo el IPE-1 mensual para encontrar las ocurrencias de eventos secos y húmedos durante todo el periodo de estudio. También se seleccionaron como objeto de estudio relevante los periodos: de agosto de 1986 a febrero de 1988, en los cuales se registraron mayores eventos secos y el fenómeno de El Niño por más tiempo; de junio de 2009 a mayo de 2010 y mayo de 1995, cuando se registraron eventos de extrema sequía; y octubre de 1998 por el huracán Mitch.

4. Los datos se ajustaron a una distribución de probabilidad gamma que se transforma a una distribución normal estandarizada (media 0 y varianza 1), procedimiento que se hizo utilizando el software spi_ls_6, desarrollado por el Centro Nacional de Mitigación de la Sequía de la Universidad de Nebraska en Estados Unidos; este software calcula el IPE en diversas escalas de tiempo, según corresponda, por ejemplo: 1, 3,6, 9 y 12 meses.

5. Finalmente, se desarrollaron los gráficos en SPSS y Microsoft Excel representando los diversos análisis de sequía para cada estación meteorológica, probabilidad de ocurrencia de sequía y se generaron, con el software ArcGIS, los mapas geoespaciales en donde se visualizan las épocas secas y lluviosas a lo largo del tiempo (ver figura 1).

Figura 1. Metodología de la estimación del IPE y representación espacial

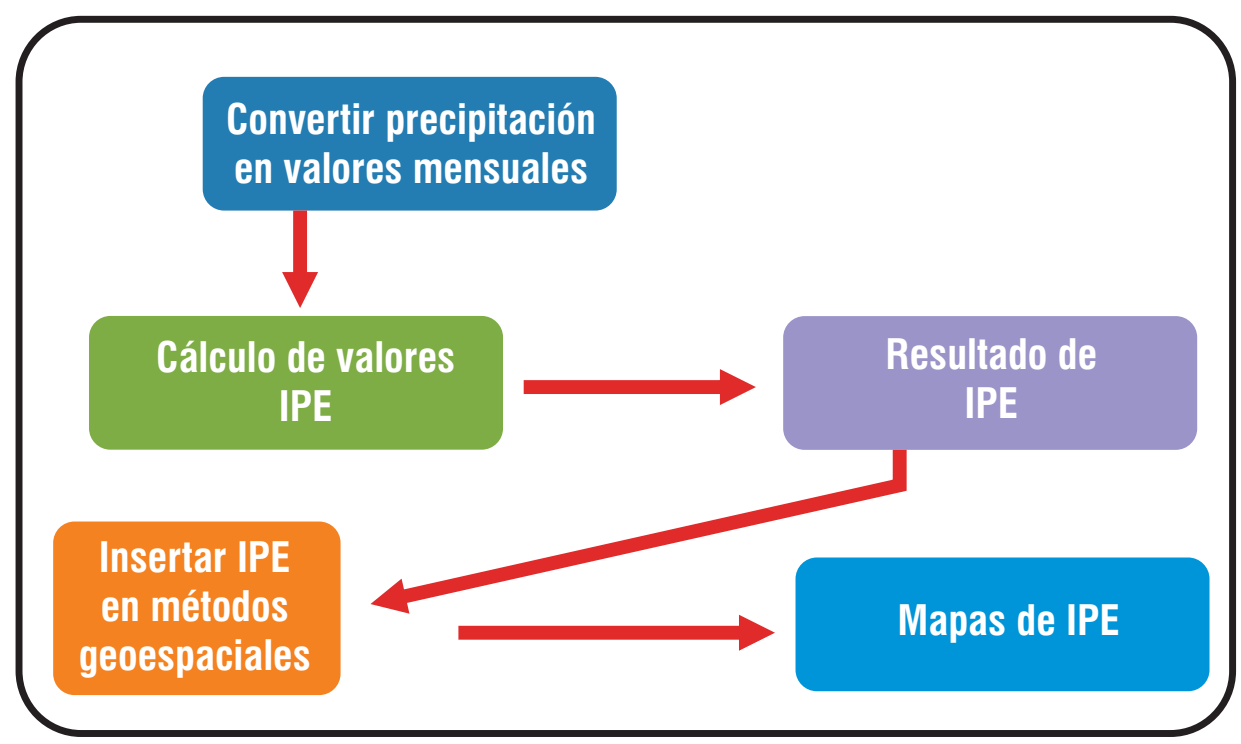

Fuente: Elaboración propia 


\section{ANÁLISIS}

Se analizaron los resultados y fueron identificados 3 períodos de severa sequía: 1986-1988, 1995, 2009-2010; porque en estos se presentó un mayor número de eventos secos para estos períodos. El cuadro 2 presenta una distribución de eventos secos, normales o húmedos, por estación meteorológica durante los años de 1975 a 2012.

Se observó que en ciertas estaciones ocurrió un mayor número de eventos secos extremos (Esparta, La Masica) y ningún evento húmedo extremo; pero, hay estaciones en donde pasó una gran mayoría de eventos extremos húmedos (La Unión, San Francisco y San Marcos) sin eventos secos extremos; mientras que Tela mantiene un equilibrio en la cantidad de eventos presentados. Como era de esperarse, se mantiene una cantidad superior en el rango de la normalidad (evento casi normal) para todas las otras estaciones.

Como consecuencia del análisis de la distribución de eventos secos, normales 0 húmedos en la zona, en el cuadro 2 se presenta la relación de los eventos secos y la presencia del fenómeno de El Niño en nuestro país desde 1975 hasta 2012. Se aprecia en dicho cuadro la relación que existe entre los años El Niño y los años marcados como secos, que es cuando la cantidad de eventos secos superan a la cantidad de eventos húmedos.

Al respecto, es preciso recordar que el fenómeno El Niño provoca una disminución de las lluvias durante casi toda la temporada lluviosa, especialmente en los meses de agosto y octubre, cuando donde el déficit de lluvia fácilmente supera el $60 \%$. Si el evento es débil llueve temprano en abril y mayo, en el sur; pero cuando es moderado llueve más en junio (Argeñal, 2010).

Cuadro 2. Relación de los períodos secos y húmedos con el fenómeno El Niño

\begin{tabular}{|c|l|c|c|c|}
\hline \multirow{2}{*}{ Año } & Descripción & \multirow{2}{*}{ Fenómeno } & \multicolumn{2}{|c|}{ Cantidad eventos } \\
\cline { 4 - 5 } & & & Secos & Húmedos \\
\hline 1975 & Seco & -- & 14 & 0 \\
\hline 1976 & Húmedo & El Niño & 1 & 2 \\
\hline 1977 & Húmedo & El Niño & 1 & 25 \\
\hline 1978 & Húmedo & & 4 & 15 \\
1979 & Húmedo & El Niño & 0 & 22 \\
\hline 1980 & Húmedo & & 0 & 24 \\
\hline
\end{tabular}




\begin{tabular}{|c|l|c|c|c|}
\hline \multirow{2}{*}{ Año } & Descripción & \multirow{2}{*}{ Fenómeno } & \multicolumn{2}{|c|}{ Cantidad eventos } \\
\cline { 4 - 5 } & & & Secos & Húmedos \\
\hline 1981 & Húmedo & & 0 & 25 \\
\hline 1982 & Húmedo & El Niño & 1 & 1 \\
\hline 1983 & Seco & El Niño & 12 & 0 \\
\hline 1984 & Seco & & 11 & 0 \\
\hline 1985 & Seco & El Niño & 20 & 0 \\
\hline 1986 & Seco & El Niño & 11 & 0 \\
\hline 1987 & Seco & El Niño & 42 & 0 \\
\hline 1988 & Húmedo & & 0 & 24 \\
\hline 1989 & Húmedo & & 3 & 4 \\
1990 & Seco & & 12 & 7 \\
\hline 1991 & Seco & El Niño & 9 & 6 \\
\hline 1992 & Seco & El Niño & 6 & 0 \\
\hline 1993 & Seco & & 20 & 1 \\
\hline 1994 & Húmedo & & 4 & 5 \\
\hline 1995 & Seco & & 43 & 0 \\
\hline 1996 & Húmedo & & 12 & 24 \\
\hline
\end{tabular}

Fuente: Elaboracion propia con base en la clasificación según valores del índice de precipitación.

\section{Máximos y mínimos}

El gráfico 1 muestra el comportamiento de máximos y mínimos en la subcuenca del río Bonito a lo largo del período de estudio 1975- 2011. Se puede observar que estas cantidades en su mayoría se encuentran en los niveles promedio esperados de -1 a 1, por tanto, la zona estudiada presenta un comportamiento balanceado.

Es de resaltar que los niveles bajos de máximos promedio de mayor prolongación que van desde 1983 hasta 1988 y de 1991 a 1995, que fueron períodos en los cuales se presentó el fenómeno de EI Niño en la mayoría de los años.

También se puede ver que hubo períodos de mayor humedad, destacándose los años 2006 al 2008, en los que se presentó el fenómeno de La Niña en nuestro país. Tal análisis se respalda con la presencia de 79 eventos húmedos por tan solo 6 eventos secos en el período antes dicho.

Así, durante el período de 2006 a 2008, resalta sobre todo la estación de la Escuela John F. Kennedy, en el municipio de San Francisco, que tuvo promedios muy por encima de lo habitual en los niveles de precipitación normal, encontrándose un SPI-12 de +2.2 y SPI-1 de +1.1 (vergráfico 1). 
Gráfico 1. Máximos y mínimos promedio SPI-12



\section{Período 1986 -1988}

La sequía de 1986-1988 comenzó en agosto de 1986, con un pico en noviembre de 1986 y finalizó en febrero de 1988. Tal sequía coincidió con la presencia del fenómeno de El Niño y se caracterizó como uno de los períodos de sequía de mayor intensidad presentados en la subcuenca del río Bonito, reportándose 58 eventos secos con 0 eventos húmedos extremos (análisis con SPI-12).

El cuadro 3 muestra el número eventos secos y húmedos por estación durante el período, para ello se utilizó el SPI-1 para analizar el comportamiento mes a mes de la precipitación. Este cuadro también indica que el $68 \%$ de los eventos extremos fueron reportados como secos; así, esta información respalda las conclusiones de los especialistas sobre la incidencia del fenómeno de El Niño durante el período estudiado.

Cuadro 3. Distribución de eventos mes a mes con SPI-1 (agosto. 1986 a feb. 1988)

\begin{tabular}{|c|c|c|c|c|c|c|c|}
\hline Estación & $\begin{array}{c}\text { Extrema- } \\
\text { damente } \\
\text { seco }\end{array}$ & $\begin{array}{c}\text { Severa- } \\
\text { mente } \\
\text { seco }\end{array}$ & $\begin{array}{c}\text { Moderada- } \\
\text { mente } \\
\text { húmedo }\end{array}$ & $\begin{array}{c}\text { Casi } \\
\text { normal }\end{array}$ & $\begin{array}{c}\text { Modera- } \\
\text { damente } \\
\text { húmedo }\end{array}$ & $\begin{array}{c}\text { Muy } \\
\text { húmedo }\end{array}$ & $\begin{array}{c}\text { Extremada- } \\
\text { mente } \\
\text { húmedo }\end{array}$ \\
\hline Esparta & 0 & 0 & 1 & 16 & 1 & 1 & 0 \\
\hline La Masica & 1 & 2 & 1 & 14 & 1 & 0 & 0 \\
\hline La Unión & 0 & 0 & 3 & 16 & 0 & 0 & 0 \\
\hline San Francisco & 0 & 2 & 1 & 16 & 0 & 0 & 0 \\
\hline San Juan & 0 & 2 & 1 & 16 & 0 & 0 & 0 \\
\hline San Marcos & 0 & 1 & 1 & 14 & 3 & 0 & 0 \\
\hline Tela & 0 & 0 & 3 & 13 & 2 & 1 & 0 \\
\hline Suma & 1 & 7 & 11 & 105 & 7 & 2 & 0 \\
\hline
\end{tabular}

Fuente: Elaboración propia con base en la clasificación de valores de IPE (Agosto 1986-febrero 1988). 
Los meses que presentaron un mayor número de eventos extremos secos fueron noviembre de 1986, abril de 1987 hasta junio del mismo año y octubre de 1987.

El mapa 2 muestra el mes de noviembre de 1986, durante el cual se presentó una mayor sequía en la subcuenca estudiada y como se observa, hubo eventos extremos de sequía en dos estaciones: San Francisco y La Masica.

Mapa 2. Comportamiento noviembre 1986, SPI-1

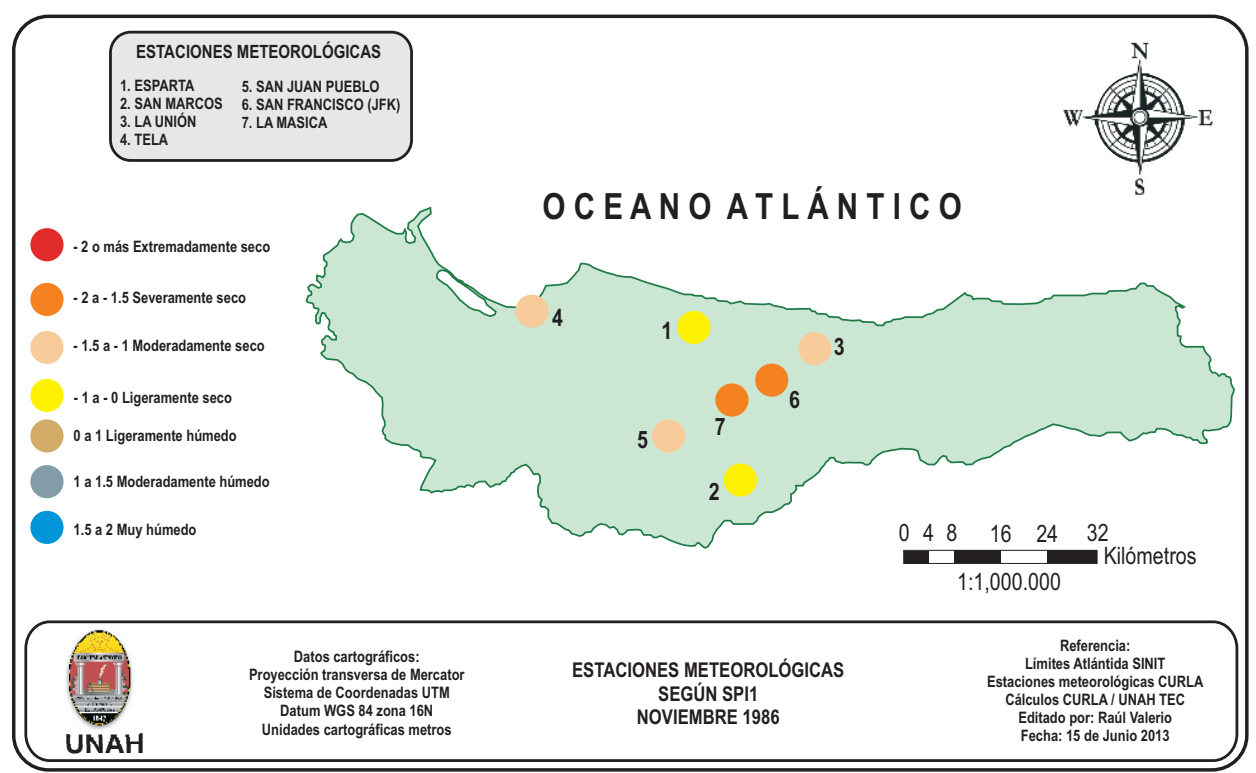

\section{Período 2009-2010}

Este período tratado fue el último durante el cual el fenómeno de El Niño azotó con mayor intensidad, según científicos estudiosos del tema (NOAA). En la zona de la subcuenca se puede apreciar su influencia, la que solo muestra información de 3 estaciones meteorológicas debido a la falta de recopilación de datos de las demás estaciones por parte de la entidad encargada.

Se hizo una subdivisión en el rango casi normal para poder apreciar de mejor manera el comportamiento de la precipitación, ya que la cantidad de eventos extremos fue mucho menor de los esperados en relación el rango de normalidad.

Durante el período en análisis se reportó una leve baja en la precipitación en los meses de junio a octubre de 2009, afectando en gran medida a la estación ubicada 
en La Unión, presentando hasta 3 eventos extremos secos (-2 o menos en SPI-1).

El mapa 3 muestra el comportamiento de la zona en el mes de junio de 2009, ya que fue el mes en donde se reportó mayor intensidad de la sequía en el período estudiado. Se observa que la estación de La Unión reveló un evento extremadamente seco, mientras que las demás estaciones se mantuvieron en el rango de normalidad de precipitación, con inclinación hacia el lado negativo de la curva normal, por tanto, este mes es considerado seco.

Mapa 3. Comportamiento en el mes de junio 2009, SPI-1

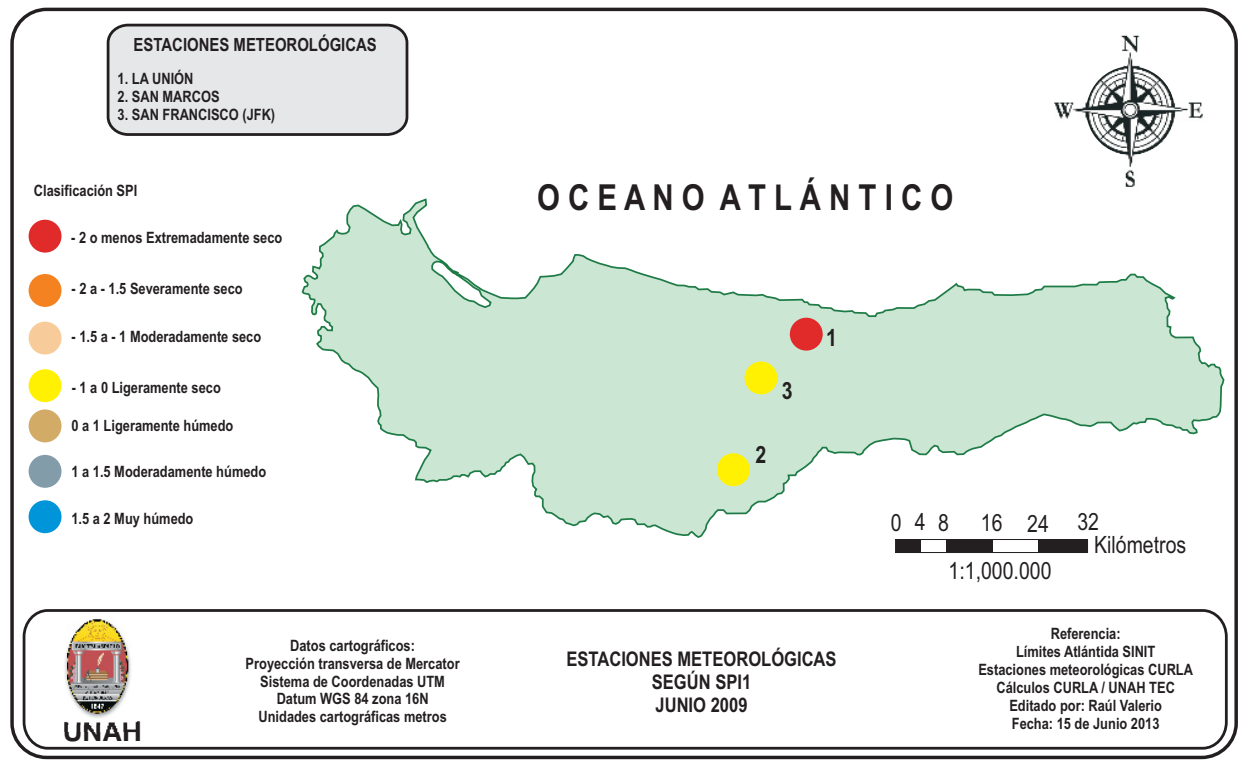

El mapa 4 describe el comportamiento de la zona en el mes de mayo del 2010, es decir, el fin del ciclo del fenómeno de El Niño en el período 2009-2010, se considera tal comportamiento utilizando el SPI-12, o sea, tomando en cuenta el análisis durante todo un año (lo que duró el fenómeno).

Como se visualiza en el mapa 4, los niveles de precipitación regresaron a sus niveles normales de precipitación luego de presentar eventos extremos secos mostrados anteriormente (mapa 3).

Es importante señalar que con el análisis del SPI-12 se encontró que la estación La Unión tuvo un promedio de -1.2 en el período estudiado, lo cual indica que las precipitaciones de lluvia fueron escasas (eventos moderadamente secos) o, mejor 
dicho, menores al comportamiento normal durante la influencia del fenómeno El Niño. Las demás estaciones reportaron un comportamiento equilibrado.

Mapa 4. Comportamiento en mayo de 2010, SPI-12

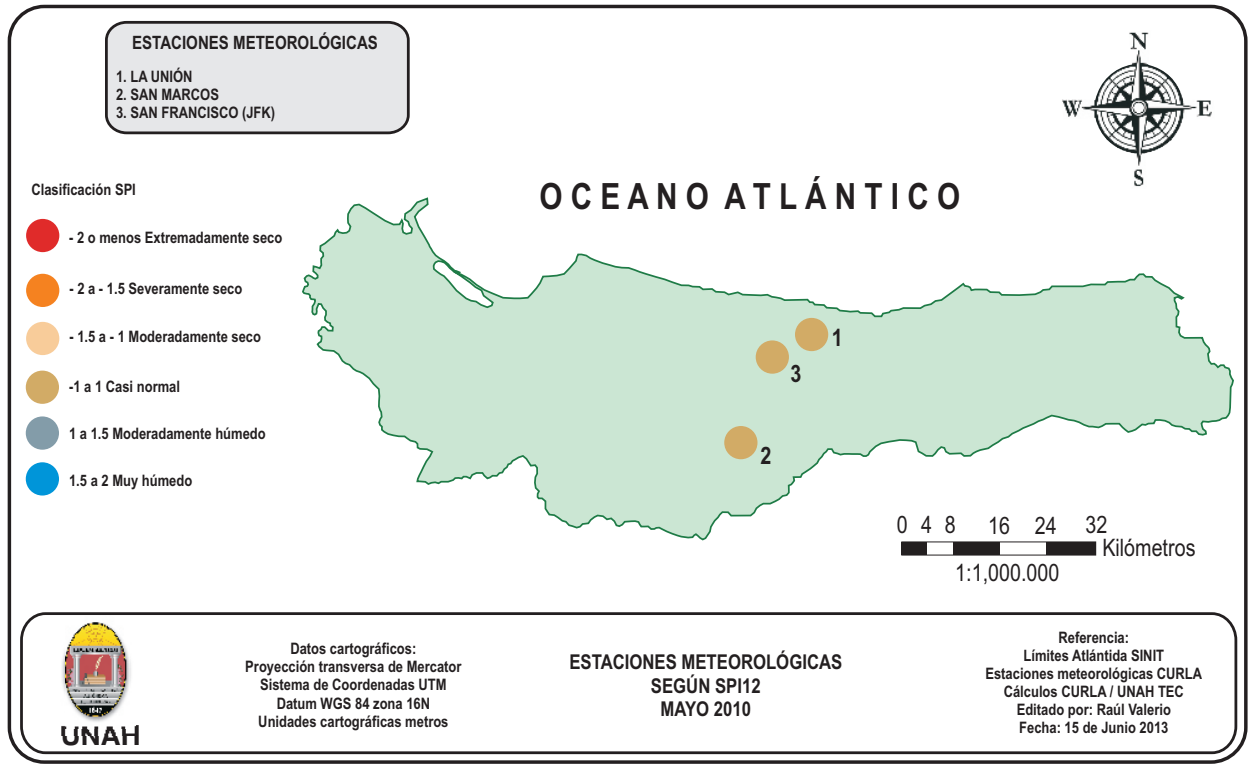

\section{Período 1995}

En el año 1995 se reportó una cantidad considerable de eventos extremos, lo cual motivó a su estudio y análisis. Así, se reportaron 43 eventos extremos secos en total, pero no se presentaron eventos húmedos, es decir, que sucedió una enorme cantidad de eventos extremos secos y por tanto es considerado seco (ver cuadro 2 ).

Como tal reporte indica que los niveles de precipitación a lo largo del año fueron menores al rango de la normalidad, se obtuvo un promedio anual del SPI-12 por debajo de -1 en casi todas las estaciones utilizadas en el estudio. Esto indica, por supuesto, el nivel de sequía presentado a lo largo de 1995.

Los meses durante los cuales se reportó un mayor número de eventos secos fueron de enero a mayo, apreciándose niveles de sequía del $67 \%$ al $100 \%$ en los eventos encontrados. La estación que manifestó más eventos secos fue la ubicada en San Juan Pueblo. Por ejemplo, en el mes de mayo de dicho año se encontró, incluso, un promedio menor a -1 en SPI-1 (ver gráfico 2). 
Gráfico 2. Porcentaje de eventos secos por mes, 1995

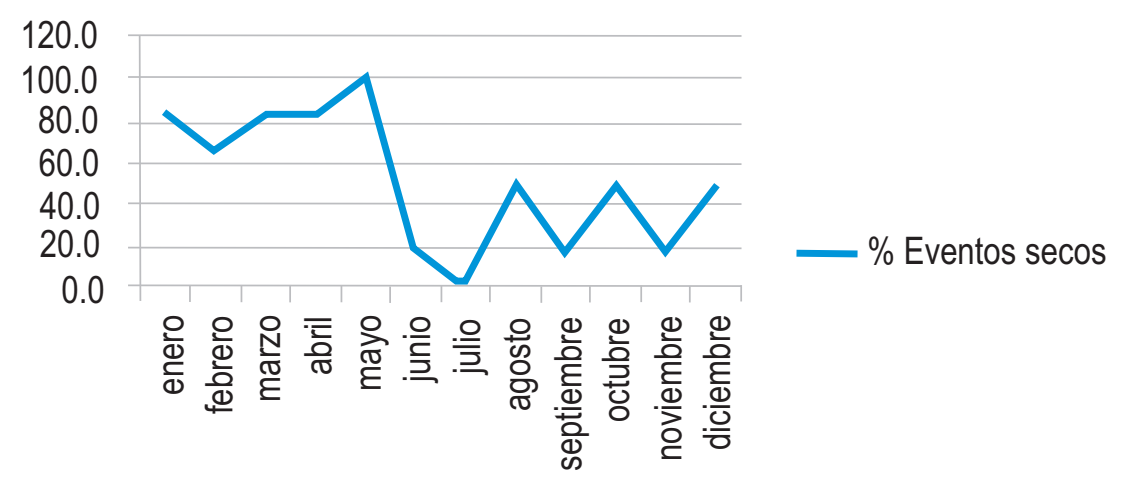

Fuente: Elaboración propia con base en valores de IPE, 1995.

El mapa 4 muestra qué eventos secos se presentaron durante el mes de mayo de 1995; además, que todas las estaciones tuvieron niveles de precipitación por debajo de lo normal, pero sobre todo realza el evento extremadamente seco en la estación de Tela.

Mapa 4. Comportamiento en mayo de 1995, SPI- 1

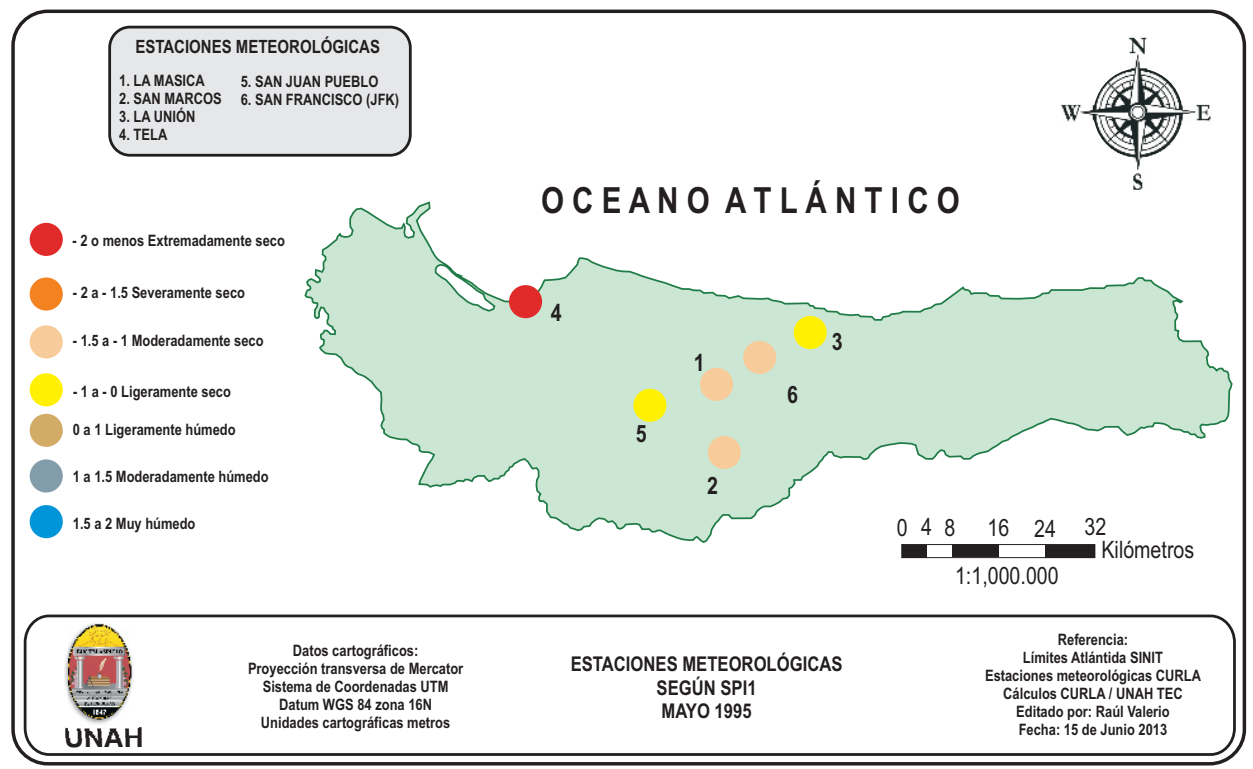




\section{Huracán Mitch}

Se analizó el comportamiento de la pluviavilidad en el mes de ocurrencia del huracán Mitch en Honduras, fenómeno ocurrido a finales de octubre e inicios de noviembre de 1998. El mapa 5 muestra tal comportamiento en ese mes utilizando el SPI-1, para así poder observar solamente la influencia de ese mes en el presente análisis.

Como era de esperarse, los resultados indican que los niveles de precipitación están sobre los niveles de promedio de manera extrema (eventos húmedos), alejados más de +1.5 desviaciones estándar, arriba del promedio en muchas de las estaciones presentes en el análisis.

Es notable considerar que en el estudio previamente mostrado en el cuadro 2, sobre el SPI-12 año a año, el año de 1998 fue seco, a pesar de tener meses como octubre muy sobre el nivel promedio de precipitación. Tal análisis es muy interesante, ya que la causa se debe a la presencia del fenómeno de El Niño en Honduras.

Mapa 5. Comportamiento en octubre de 1998, SPI- 1

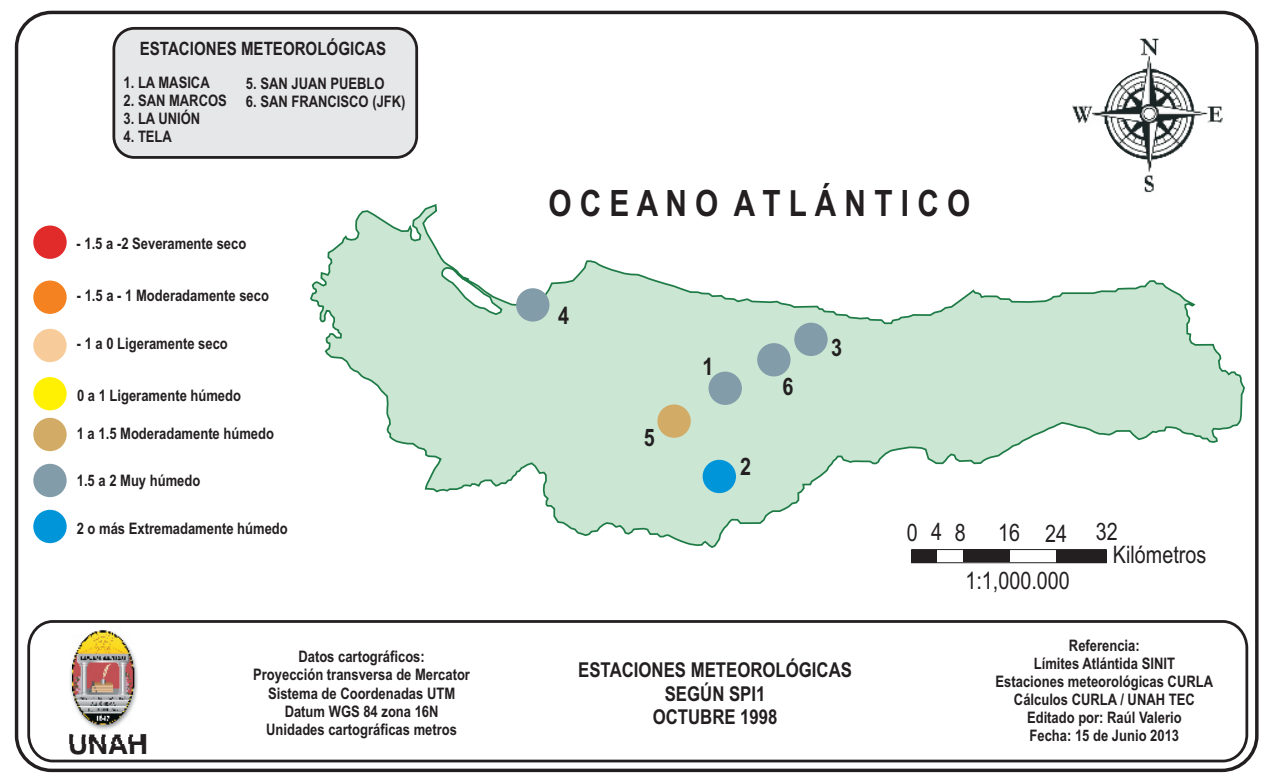




\section{CONCLUSIONES}

1. El índice de precipitación estandarizado (IPE) pudo aplicarse de manera satisfactoria en la subcuenca del río Bonito, ya que se obtuvieron resultados acorde a la situación real presentada en la zona de estudio.

2. En términos generales, la subcuenca no presenta muchos eventos extremadamente secos, a excepción de los periodos analizados anteriormente (agosto de 1986, febrero de 1988, 1995, junio de 2009 - mayo de 2010), cualquier otra situación de sequía en la zona será, por tanto, relacionada con el mal uso de los recursos naturales de los pobladores o por otro fenómeno, pero no por la ausencia de precipitación.

3. En el $60 \%$ de los casos en los cuales el fenómeno de El Niño se presentó en Honduras hubo sequías notorias. Es decir, la presencia de dicho fenómeno se hace notar.

4. La ocurrencia de eventos extremos igualmente se presentaron a lo largo del año sin importar el mes.

5. Como se refleja en el estudio del huracán Mitch, la diferencia entre el SPI-1 y el SPI-12 es notable gracias a la injerencia de este tipo de fenómenos.

6. La predicción de sequía para los años futuros no pudo lograrse debido a la falta de datos de precipitación recientes de las estaciones meteorológicas, ya que en solo 2 estaciones hubo registros del año 2012.

\section{AGRADECIMIENTOS}

Se agradece el apoyo brindado por la Dirección de Investigación Científica y Posgrado de la UNAH, por el financiamiento para realizar la investigación y la supervisión en el desarrollo de la misma.

Igualmente, gracias a las instituciones que colaboraron con esta investigación: Secretaría de Recursos Naturales, Servicio Meteorológico Nacional, Instituto de Ciencias de la Tierra; también a la máster Claudia Lardizábal y a la licenciada Tania Peña, quienes dieron su apoyo a los investigadores.

\section{BIBLIOGRAFÍA}

Argenal, Francisco (2010). Variabilidad climática y cambio climático en Honduras. Programa de las Naciones Unidas para el Desarrollo.

Casanoves, Fernando. 2011. Estadística para ciencias agropecuarias. Argentina: Editorial Brujas.

Cancelliere, Antonino. (2006). Drougth Forecasting using the Standarized Precipitation Index. Italia: Springer Science. 
Edwards, Daniel and McKee, Thomas (1997). Characteristics of 20th century drought in the United States at multiple time scales. Climatology Report, 97, 2, Colorado State University.

Guttman, Nathaniel. (1999). Accepting the Standardized Precipitation Index: A Calculation Algorithm. Paper No.97156, Journal of the American Water Resources Association, Carolina del Norte.

Hayes, Michael Svoboda; Mark, David; Wilhite, Don y Vanyarkho, Olga. (1999). Monitoring the 1996 Drought Using the Standardized Precipitation Index. Bulletin of the American Meteorological Society, 80, 429-438.

Hernández, Alexander Javier. (2003). Dinámica del uso de la tierra y de la oferta y la demanda hídrica en la Cuenca del río Guacerique. Tesis de Maestría, CATIE, Costa Rica.

Jiménez, Francisco. (2007). Practica de análisis de vulnerabilidad. Turrialba: CATIE. Jiménez, Francisco. (2007). Curso gestión del riesgo a desastres. Turrialba: CATIE.

Lardizábal, Claudia. (2008). Análisis de la sequía y alternativas de adaptación y mitigación en la subcuenca del río Guacerique. Tesis de Maestría, CATIE, Costa Rica.

Lugo, Hubp; Inbar, José y Moshe. (2002). Desastres naturales en América Latina. México: Fondo de Cultura Económica.

McKee, Thomas; Doesken, Nolan and Kleist, John. (1993). The relationship of drought frequency and duration to time scale. In Proceedings of the Eighth Conference on Applied Climatology. Anaheim, California, 17-22 January 1993. Boston:America Meteorological Society.

INYPSA. (2011). Diagnóstico integral multidimensional de La Ceiba. Elaboración de planes y ordenamiento territorial de la subregión IV, Valle de Lean. La Ceiba. Proyecto de gestión sostenible de los recursos naturales y cuencas del corredor biológico mesoamericano en el Atlántico hondureño.

World Metereological Organization. (2012). Standarized precipitation Index User Guide. Switzerland: Chair Publications Board. 